\title{
Probing Deconfinement with Polyakov Loop Susceptibilities
}

\author{
Pok Man Lo, ${ }^{1}$ Bengt Friman, ${ }^{1}$ Olaf Kaczmarek, ${ }^{2}$ Krzysztof Redlich, ${ }^{3,4}$ and Chihiro Sasaki ${ }^{5}$ \\ ${ }^{1}$ GSI, Helmholzzentrum für Schwerionenforschung, Planckstr. 1, D-64291 Darmstadt, Germany \\ ${ }^{2}$ Fakultät für Physik, Universität Bielefeld, 33615 Bielefeld, Germany \\ ${ }^{3}$ Institute of Theoretical Physics, University of Wroclaw, PL-50204 Wroctaw, Poland \\ ${ }^{4}$ Extreme Matter Institute EMMI, GSI, Planckstr. 1, D-64291 Darmstadt, Germany \\ ${ }^{5}$ Frankfurt Institute for Advanced Studies, D-60438 Frankfurt am Main, Germany
}

(Dated: November 5, 2021)

\begin{abstract}
The susceptibilities of the real and imaginary parts, as well as of the modulus of the Polyakov loop, are computed in $S U(3)$ lattice gauge theory. We show that the ratios of these susceptibilities are excellent probes of the deconfinement transition, independent of the renormalization of the Polyakov loop and only weakly dependent on the system size. The ratios are almost temperature independent above and below the transition and exhibit a discontinuity at the transition temperature. This characteristic behavior can be understood in terms of the global $\mathcal{Z}_{3}$ symmetry of the Yang-Mills Lagrangian and the general properties of the Polyakov loop probability distribution.
\end{abstract}

PACS numbers: 25.75.Nq, 11.15.Ha, 24.60.-k, 05.70.Jk

Systems described by a pure $S U\left(N_{c}\right)$ gauge theory in $(d+1)$-dimensions undergo a phase transition at finite temperature. Owing to the conjectured universality with d-dimensional $\mathcal{Z}_{N_{c}}$ spin systems [1], this transition is of general interest.

For $N_{c}=3$, the transition is first order and is characterized by spontaneous breaking of the global $\mathcal{Z}_{3}$ center symmetry of the Yang-Mills Lagrangian [2 6 .

The Polyakov loop, which is linked to the free energy of a static quark immersed in a hot gluonic medium [7, [8], can be used to define an order parameter of the deconfinement transition. At low temperatures its thermal expectation value vanishes, implying color confinement, while at high temperatures it is non-zero, resulting in a finite energy of a static quark and consequently deconfinement of color. While the basic thermodynamic functions of $S U(3)$ pure gauge theory are well established within the lattice approach [2 [6, 9 12], the situation is less satisfactory for the renormalized Polyakov loop and, in particular, for the corresponding susceptibilities.

In a pure $S U(3)$ gauge theory, the temperature of the confinement-deconfinement transition is uniquely defined by the discontinuity of the order parameter, since the transition is first order. More generally, for systems where the transition is continuous, e.g. QCD, the transition temperature is identified by a maximum of the fluctuations, quantified e.g. by one of the Polyakov loop susceptibilities. For $N_{c} \geq 3$, the Polyakov loop operator is complex valued. Correspondingly, one can define susceptibilities of the real and imaginary parts as well as of the modulus of the Polyakov loop.

In a Yang-Mills theory, formulated on the lattice, the ultraviolet divergence of the bare quark-antiquark free energy implies that, in the continuum limit, the bare Polyakov loop vanishes at any temperature. Thus, in order to obtain a physically meaningful continuum limit, the Polyakov loop must be renormalized [13, 14]. The renormalization of gluon correlation functions in general, and the Polyakov loop susceptibility in particular, are still subject to uncertainties.

In this paper we bypass these ambiguities by considering the ratios of Polyakov loop susceptibilities. In particular, we focus on their properties near the deconfinement transition. To this end, we compute the temperature dependence of the Polyakov loop susceptibilities within $\mathrm{SU}(3)$ lattice gauge theory on different sized lattices and examine the relevance of susceptibility ratios as probes of the deconfinement transition.

We argue that these characteristics are naturally understood in terms of the global $\mathcal{Z}_{3}$ symmetry and general properties of the Polyakov loop probability distribution. Moreover, they are independent of the renormalization of the Polyakov loop and depend only weakly on the volume. This implies that the susceptibility ratios are excellent observables for identifying the confinementdeconfinement phase transition in $S U(3)$ pure gauge theory.

The Polyakov loop susceptibilities on the lattice. On a $N_{\sigma}^{3} \times N_{\tau}$ lattice, the Polyakov loop is defined as the trace of the product over temporal gauge links,

$$
\begin{array}{r}
L_{\vec{x}}^{\text {bare }}=\frac{1}{N_{c}} \operatorname{Tr} \prod_{\tau=1}^{N_{\tau}} U_{(\vec{x}, \tau), 4}, \\
L^{\text {bare }}=\frac{1}{N_{\sigma}^{3}} \sum_{\vec{x}} L_{\vec{x}}^{\text {bare }} .
\end{array}
$$

Due to the $\mathcal{Z}_{3}$ symmetry of the pure gauge action, this quantity vanishes, when averaged over all gauge field configurations. Furthermore the Polyakov loop is strongly $N_{\tau}$ dependent and must be renormalized.

These problems are avoided by considering the renormalized Polyakov loop [13],

$$
L^{\text {ren }}=\left(Z\left(g^{2}\right)\right)^{N_{\tau}} L^{\text {bare }}
$$

and introducing the ensemble average of the modulus thereof, $\left\langle\left|L^{\text {ren }}\right|\right\rangle$. The latter is well defined in the con- 


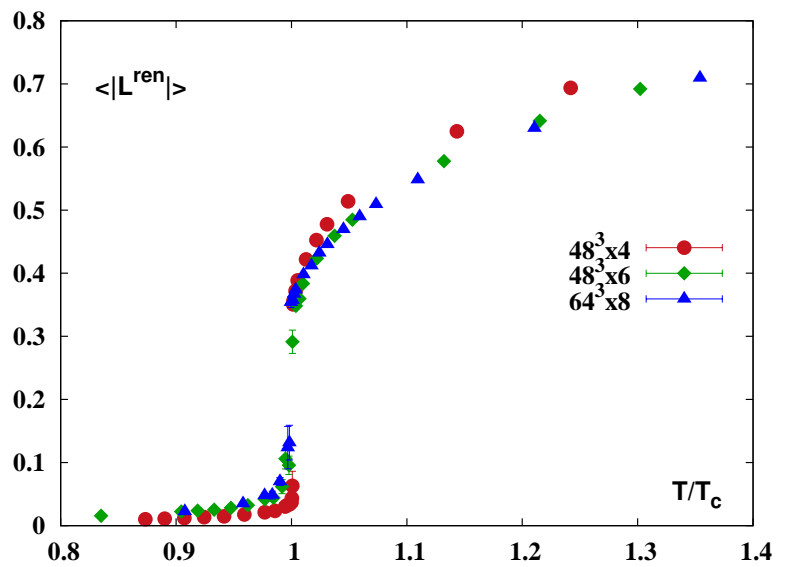

FIG. 1: The modulus of renormalized Polyakov loop $\left\langle\left|L^{\text {ren }}\right|\right\rangle$ obtained in $S U(3)$ lattice gauge theory.

tinuum and thermodynamic limits and is an order parameter for the spontaneous breaking of the $\mathcal{Z}_{3}$ center symmetry. The lattice gauge theory result for $\left\langle\left|L^{\text {ren }}\right|\right\rangle$, as a function of temperature, is shown in Fig. 1

As noted above, the location of the phase transition is correlated with a maximum (or divergence) of the fluctuations of the order parameter. For the confinementdeconfinement transition, these fluctuations are reflected in the renormalized Polyakov loop susceptibility ${ }^{1}$

$$
T^{3} \chi_{A}=\frac{N_{\sigma}^{3}}{N_{\tau}^{3}}\left(\left\langle|L|^{2}\right\rangle-\langle|L|\rangle^{2}\right) .
$$

In the $S U(3)$ gauge theory, the Polyakov loop operator is complex. Consequently, in addition to $\chi_{A}$, one can also explore independent fluctuations of the real and imaginary parts of the Polyakov loop. Taking the $\mathcal{Z}_{3}$ symmetry into account, we define a longitudinal and a transverse susceptibility ${ }^{2}$

$$
\begin{aligned}
& T^{3} \chi_{L}=\frac{N_{\sigma}^{3}}{N_{\tau}^{3}}\left[\left\langle\left(L_{L}\right)^{2}\right\rangle-\left\langle L_{L}\right\rangle^{2}\right] . \\
& T^{3} \chi_{T}=\frac{N_{\sigma}^{3}}{N_{\tau}^{3}}\left[\left\langle\left(L_{T}\right)^{2}\right\rangle-\left\langle L_{T}\right\rangle^{2}\right],
\end{aligned}
$$

where $L_{L}=\operatorname{Re}(\tilde{L})$ and $L_{T}=\operatorname{Im}(\tilde{L})$. Here we have introduced the $\mathcal{Z}_{3}$ transformed Polyakov loop, $\tilde{L}=L e^{2 \pi n i / 3}$, with $n=0, \pm 1$. The phase of the transformation is chosen such that for $T>T_{c}$ the expectation value of the transformed Polyakov loop, $\langle\tilde{L}\rangle$, is real. For $T<T_{c}$ the expectation value of the Polyakov loop vanishes, and we take $n=0$. Thus, in the latter case $L_{L}=\operatorname{Re}(L)$ and $L_{T}=\operatorname{Im}(L)$.

\footnotetext{
${ }^{1}$ In the following we deal only with the renormalized Polyakov loop, and hence drop the superscript on $L^{\text {ren }}$.

2 There is no mixing between longitudinal and transverse susceptibilities.
}

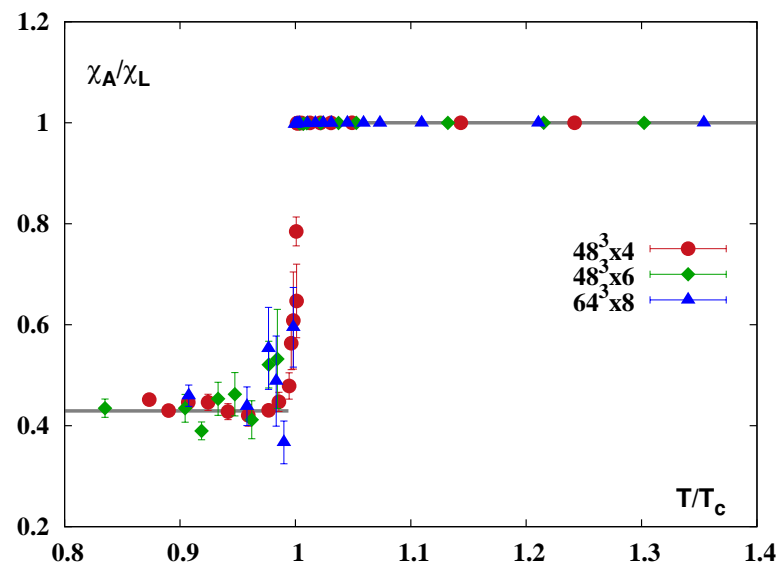

FIG. 2: The ratio of the modulus $\chi_{A}$ and longitudinal $\chi_{L}$ Polyakov loop susceptibilities obtained in $S U(3)$ lattice gauge theory. The lines shows $\chi_{A} / \chi_{L}=1$ for $T>T_{c}$ and $\chi_{A} / \chi_{L}=$ $2-\pi / 2$ for $T<T_{c}$ (see text).

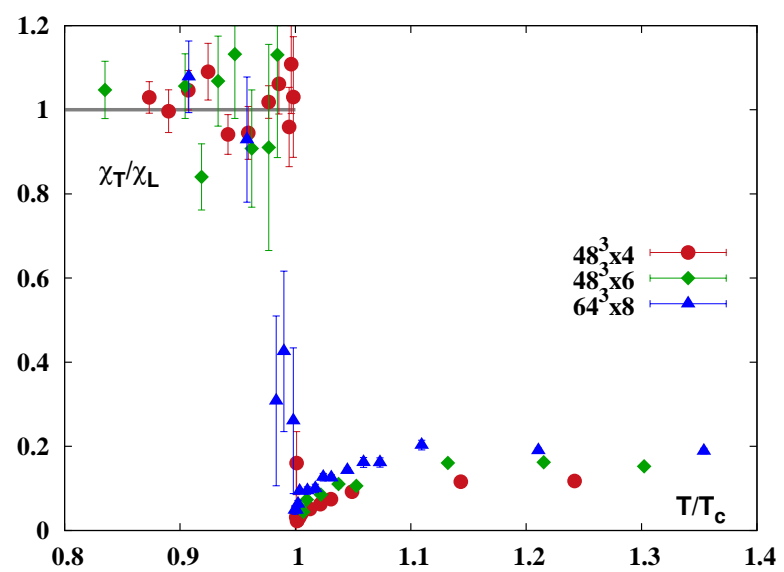

FIG. 3: The ratio of the transverse $\chi_{T}$ and longitudinal $\chi_{L}$ Polyakov loop susceptibilities. The line shows $\chi_{T} / \chi_{L}=1$ for $T<T_{c}$ (see text).

We have computed the Polyakov loop susceptibilities, Eqs. (44), (5) and (6), within $S U(3)$ lattice gauge theory, using the (1,2)-tree-level Symanzik improved gauge action on a $N_{\sigma}^{3} \times N_{\tau}$ lattice. We consider lattices of temporal size $N_{\tau}=4,6$ and 8 and spatial extent $N_{\sigma}$ varying from 16 to 64 . However, in order to make the figures more transparent, we show results only for the largest volumes, i.e. for $N_{\sigma}=48$ and 64 . We set the temperature for the three temporal lattice extents by varying the bare coupling and use the temperature scale determined by the zero temperature string tension, as well as the critical couplings of the deconfinement transition [10, 15]. The gauge field configurations were generated using one heatbath and four overrelaxation updates per sweep with 15000 sweeps in general and up to 100000 sweeps close to the critical temperature, $T_{c}$. 
The ratios of susceptibilities. In Figs. 2 and 3 we show $S U(3)$ lattice gauge theory results for the ratios $R_{A}=$ $\chi_{A} / \chi_{L}$ and $R_{T}=\chi_{T} / \chi_{L}$, as functions of temperature. Since renormalization as well as volume and temperature factors cancel in these ratios, they provide robust probes of the deconfinement transition. Indeed, both ratios exhibit a strong discontinuity at the deconfinement phase transition and are almost temperature and volume independent. A straightforward interpretation of the properties of $R_{A}$ and $R_{T}$ is obtained by using general considerations and the $\mathcal{Z}_{3}$ center symmetry.

Consider first the ratio $R_{T}$ for $T<T_{c}$. In the $\mathcal{Z}_{3}$ symmetric phase, the expectation value of any symmetry breaking operator, e.g. $\tilde{L}$ or $\tilde{L}^{2}$, must vanish. Hence,

$$
V\left(\left\langle\tilde{L}^{2}\right\rangle-\langle\tilde{L}\rangle^{2}\right)=\chi_{L}-\chi_{T}=0,
$$

which implies that $\chi_{L}=\chi_{T}$. Since $\chi_{L}$ and $\chi_{T}$ are both non-zero, it follows that $R_{T}=1$, as shown in Fig. 3 ,

The fact that $R_{A} \simeq 1$ in the deconfined phase, as shown in Fig. 2, follows from the following argument. In the broken symmetry phase, we introduce shifted operators $\delta L_{L}$ and $\delta L_{T}$ :

$$
\begin{aligned}
& L_{L}=L_{0}+\delta L_{L}, \\
& L_{T}=\delta L_{T},
\end{aligned}
$$

where $L_{0}=\langle\tilde{L}\rangle=\left\langle L_{L}\right\rangle$ is the (real) expectation value of the transformed Polyakov loop and the shifted operators $\delta L_{i}$, with $i=(L, T)$, describe the fluctuations about the mean. The thermal average of the shifted operator squared yields the corresponding susceptibility, $V\left\langle\left(\delta L_{i}\right)^{2}\right\rangle=\chi_{i}, i=L, T$. We then expand the modulus of the Polyakov loop $|L|=|\tilde{L}|$ in the shifted operators,

$$
\begin{aligned}
|L| & =\sqrt{L_{L}^{2}+L_{T}^{2}} \\
& \approx L_{0}\left(1+\frac{\delta L_{L}}{L_{0}}+\frac{1}{2} \frac{\left(\delta L_{T}\right)^{2}}{L_{0}^{2}}\right) .
\end{aligned}
$$

Using the fact that by definition $\left\langle\delta L_{i}\right\rangle=0$, we find

$$
\langle|L|\rangle \simeq L_{0}\left(1+\frac{1}{2} \frac{\left\langle\left(\delta L_{T}\right)^{2}\right\rangle}{L_{0}^{2}}\right)
$$

while

$$
\left\langle|L|^{2}\right\rangle=L_{0}^{2}\left(1+\frac{\left\langle\left(\delta L_{T}\right)^{2}\right\rangle}{L_{0}^{2}}+\frac{\left\langle\left(\delta L_{L}\right)^{2}\right\rangle}{L_{0}^{2}}\right) .
$$

This implies that to leading order in the expansion of $|L|, \chi_{A} \simeq \chi_{L}$ and hence $R_{A} \simeq 1$ for $T>T_{c}$, as shown in Fig. 2

The properties of $R_{T}$ in the deconfined and $R_{A}$ in the confined phase cannot be directly linked to the center symmetry. Using Eq. (11), we find that

$$
\chi_{T} \simeq V\left(\langle|L|\rangle^{2}-\left\langle L_{L}\right\rangle^{2}\right) .
$$

Thus, in general, $\chi_{T}$ can be non-vanishing in the $\mathcal{Z}_{3}$ broken phase. However, its value in the high-temperature phase is not constrained by symmetries or general principles.

In Fig. 3 we show, that above the phase transition, $\chi_{T}$ is in fact much smaller than $\chi_{L}$. In the temperature range considered, we find that, for $T>T_{c}$, the ratio $R_{T}$ is weakly dependent on the temperature and does not exceed $\simeq 0.2$. It has been argued [16, 17] that in the broken $\mathcal{Z}_{3}$ symmetry phase of the $S U(3)$ gauge theory, $R_{T}$ can be as large as 0.4. We note that in our results, a dependence of $R_{T}$ on $N_{\tau}$ remains. Hence, we cannot at present draw firm conclusions on the continuum extrapolation of this quantity.

Finally, we turn to the value of $R_{A}$ in the confined phase. In Fig. 2 we show that, for $T<T_{c}, R_{A}$ is approximately temperature independent, with the lattice results clustering around a value slightly larger than 0.4. This property of $R_{A}$ can be understood by assuming that in the symmetric phase, the probability distribution for the Polyakov loop is, to a good approximation, Gaussian, with the partition function ${ }^{3}$

$$
Z=\int d L_{L} d L_{T} e^{-V T^{3}\left[\alpha(T)\left(L_{L}^{2}+L_{T}^{2}\right)\right]},
$$

where the integrations extend from $-\infty$ to $\infty$. The susceptibilities are then obtained by performing elementary integrals

$$
\begin{aligned}
& \chi_{L}=\frac{1}{2 \alpha T^{3}}, \quad \chi_{T}=\frac{1}{2 \alpha T^{3}}, \\
& \chi_{A}=\frac{1}{2 \alpha T^{3}}\left(2-\frac{\pi}{2}\right) .
\end{aligned}
$$

Consequently, $R_{A}=(2-\pi / 2) \simeq 0.429$, in good agreement with the lattice results, shown in Fig. 2, We note that the Gaussian approximation is not expected to be valid close to $T_{c}$, where the coefficient $\alpha(T)$ in (14) is small and hence higher order terms cannot be neglected.

In $S U(2)$ gauge theory the Polyakov loop is real, so the corresponding integrals are one-dimensional, which implies a slightly different ratio, $R_{A}^{S U(2)}=(1-2 / \pi) \simeq 0.363$. This value is indeed in agreement with lattice results for the $S U(2)$ Polyakov loop susceptibilities below $T_{c}$, outside of the critical region [18]. These results indicate that in the symmetric phase, the effective Polyakov loop potential is well approximated by a Gaussian both in $S U(2)$ and $S U(3)$ lattice gauge theories.

Conclusions. We have shown, that the ratios of Polyakov loop susceptibilities provide an excellent signal for the deconfinement phase transition in $S U(3)$ gauge theory. The ratios are discontinuous at the phase transition and only weakly temperature dependent on either side of $T_{c}$. Moreover, they are independent of the Polyakov loop

\footnotetext{
${ }^{3}$ More precisely, the quadratic terms of the effective action are responsible for the dominant contribution to the Polyakov loop susceptibility. However, higher order, non-Gaussian terms are decisive for the determination of higher order cumulants.
} 
renormalization and only weakly dependent on the system size.

We have also shown that, with one exception, the ratios obtained outside of the transition region, can be understood in terms of general symmetry arguments and the observation, that in the confined phase, the Polyakov loop probability distribution is well approximated by a Gaussian. There is, however, no restriction by symmetry on the ratio of the transverse to longitudinal susceptibility, $\chi_{T} / \chi_{L}$ in the deconfined phase. We find that, above $T_{c}$ this ratio is fairly small and varies weakly with temperature.

In QCD, the global $\mathcal{Z}_{3}$ symmetry is explicitly broken by finite quark masses. Hence, the properties of the susceptibility ratios in QCD can differ from those in pure gauge theory. In particular, the discontinuity will most likely be smoothened, since in QCD the transition is continuous. Nevertheless, outside of the transition region, the ratios may approximately reflect the constraints from center symmetry and thus provide a useful probe of the confinement-deconfinement transition also in full QCD.

\section{Acknowledgments}

We acknowledge stimulating discussions with Frithjof Karsch. P.M.Lo acknowledges the support of the Frankfurt Institute for Advanced Studies (FIAS). B.F. is supported in part by the Extreme Matter Institute EMMI. K.R. acknowledges partial support of the Polish Ministry of National Education (NCN). The work of C.S. has been partly supported by the Hessian LOEWE initiative through the Helmholtz International Center for FAIR (HIC for FAIR). The numerical calculations have been performed on the Bielefeld GPU Cluster.
[1] B. Svetitsky, and L. G. Yaffe, Nucl. Phys. B 210 (1982) 423.

[2] L. G. Yaffe, and B. Svetitsky, Phys. Rev. D 26 (1982) 963.

[3] G. Boyd, J. Engels, F. Karsch, E. Laermann, C. Legeland, M. Lutgemeier, and B. Petersson, Phys. Rev. Lett. 75 (1995) 4169

[4] G. Boyd, J. Engels, F. Karsch, E. Laermann, C. Legeland, M. Lutgemeier, and B. Petersson, Nucl. Phys. B 469 (1996) 419.

[5] M. Fukugita, M. Okawa, and A. Ukawa, Nucl. Phys. B 337 (1990) 181.

[6] S. Borsanyi, G. Endrodi, Z. Fodor, S. D. Katz, and K. K. Szabo, JHEP 1207 (2012) 056.

[7] L. D. McLerran, and B. Svetitsky, Phys. Lett. B 98 (1981) 195. L. D. McLerran, and B. Svetitsky, Phys. Rev. D 24 (1981) 450.

[8] A. M. Polyakov, Phys. Lett.B 72 (1978) 477. G.t Hooft, Nucl. Phys. B 138 (1978) 1. L. Susskind, Phys.Rev.D 20 (1979) 2610.

[9] Y. Iwasaki et al., Phys. Rev. D 46 (1992) 4657.
[10] R. G. Edwards, U. M. Heller, and T. R. Klassen, Nucl. Phys. B 517 (1998) 377.

[11] G. Boyd, J. Engels, F. Karsch, E. Laermann, C. Legeland, M. Lutgemeier, and B. Petersson, Phys. Rev. Lett. 75 (1995) 4169; Nucl. Phys. B 469 (1996) 419.

[12] T. DeGrand et al., Nucl. Phys. B 454 (1995) 615.

[13] O. Kaczmarek, F. Karsch, P. Petreczky, and F. Zantow, Phys. Lett. B 543 (2002) 41.

[14] U. M. Heller, and F. Karsch, Nucl. Phys. B 251 (1985) 254. R. V. Gavai, Phys. Lett. B 691 (2010) 146. S. Gupta, K. Huebner, and O. Kaczmarek, Phys. Rev. D 77 (2008) 034503.

[15] B. Beinlich, F. Karsch, E. Laermann and A. Peikert, Eur. Phys. J. C 6 (1999) 133.

[16] A. Dumitru, Y. Hatta, J. Lenaghan, K. Orginos, and R. D. Pisarski, Phys. Rev. D 70 (2004) 034511.

[17] A. Dumitru, and R. D. Pisarski, Phys. Rev. D 66 (2002) 096003.

[18] J. Engels, and T. Scheideler, Nucl. Phys. B 539 (1999) 557. 